\title{
Density functional theoretical studies on photoswitching and charge migration dynamics of thio and selenoureidopeptides
}

\author{
Sherin Joy I Vommina V Sureshbabu | Ganga Periyasamy
}

Department of Chemistry, Bangalore University, Bangalore, Karnataka, India

\section{Correspondence}

Ganga Periyasamy, Department of Chemistry, Bangalore University, Bangalore, Karnataka 560001, India.

Email: ganga.periyasamy@gmail.com

\begin{abstract}
Density functional theoretical calculations have been performed to investigate the changes in electronic structure at ground and excited states of ureidopeptides on substitution with higher chalcogens like sulphur and selenium for oxygen. This replacement results in a reduced preference towards the intramolecular hydrogen bonding interaction, thus linear conformers are found to be stable at both states. Nevertheless, conformational switching observed during this process is mainly due to $\mathrm{n}$ to $\pi^{*}$ transition that leads to the dihedral angle $\omega$ change from trans to cis with a rotational barrier of 10 to $17 \mathrm{kcal} \mathrm{mol}^{-1}$. The computed barrier is lesser than that reported for oxopeptides $\left(20 \mathrm{kcal} \mathrm{mol}^{-1}\right)$. And, the hole migration dynamics after immediate ionization illustrates that the hole originated at ureido end evolves in time (2-4 fs) while the hole generated at the carboxylate end will not evolve as reported for ureidopeptides. The usage of these candidates as photoswitches has also been explored.
\end{abstract}

\section{KEYWORDS}

charge transfer, DFT, photoswitch, selenoureidopeptides, thioureidopeptides

\section{1 | INTRODUCTION}

The introduction of photoswitchable elements in peptides ${ }^{[1]}$ are interesting, which gives control over their structure and bioactivities such as catalysis, protein folding, receptorligand interaction, and ion-channel gating. ${ }^{[2]}$ The switchable nature has a profound effect in increasing their prosperity in advanced technologies, which include reversible biosensors, photoactivated medicines, and molecular devices. ${ }^{[3]}$ To date, many biological photoswitches based on azobenzene, spiropyrene-centred peptidomimetics have been explored both in vivo and in vitro. ${ }^{[4]}$ The lack of $100 \%$ stabilization of a single conformer in these systems encourages the search for new molecules with higher efficiency. The major drawbacks of proteins and other bio entities are their complicated structure with numerous possible conformations that lead to synthesis of simpler synthetic analogous. Whereas smaller chain peptide-based photoswitchable materials are convenient, for being exist with less number of conformers with high temporal and spatial control. ${ }^{[4]}$ Photoswitching of peptide-based materials rely on the conformational change at the peptide bond from trans to cis and vice versa in the presence of UV/vis light of suitable wavelength. ${ }^{[5]}$ The conformational switching can be tuned either by covalently modifying the peptide bonds with substituents ${ }^{[6]}$ or through the isosteric replacement of oxygen in the peptide bond by higher chalcogens. ${ }^{[2,7]}$ The latter has been proved to be advantageous over others to have a single stable conformer in the ground and excited states in oxygen-containing peptides. In this regard, we have studied in detail the photoswtiching ability of one of the peptidomimetics, ureidopeptides (UPs) with various higher chalcogen substituents.

Ureidopeptidomimetics have their peptide bonds $(-\mathrm{C}(\mathrm{O})$ $\mathrm{NH}-)$ substituted by urea $(-\mathrm{HNC}(\mathrm{O}) \mathrm{NH}-)$ and have been synthesized with various substituents on either ends of the peptide chain. ${ }^{[8-10]}$ These molecules have potential applications in the field of drug delivery, sensors, and molecular devices. ${ }^{[11,12]}$ Further, substitution of ureido group for the 
natural peptide bonds are found to reform the peptide backbone structure and tune their functions as proteins or enzymes beneficially. ${ }^{[13]}$ And this arises because of the ability of ureido group to increase the possibility of helical folding through hydrogen bonding interactions in biosystems. ${ }^{[14]}$ However, the major setback in the usage of UP is their high affinity towards water. It is due to the simultaneous hydrogen bond donor and acceptor nature of UPs. ${ }^{[15]}$ Specifically, the tendency of ureido group to form strong hydrogen bonds makes them get proteolyzed easily in the cellular protein aqueous environment. ${ }^{[16]}$ This led to the search of alternative entities that preserve the advantages of UPs simultaneously being proteolysis resistant when placed in the aqueous cellular environment.

Thus, many UP bond surrogates have been proposed where the oxygen in the carbonyl group in urea is substituted with less electronegative elements of the chalcogen group (sulphur and selenium). ${ }^{[17]}$ The substitution of oxygen in ureido peptides with sulphur and selenium produces thioureidopeptides (TUPs) and selenoureidopeptides (SUPs), respectively. Generally, peptides that contain sulphur and selenium are prone to display interesting physical, chemical and biological activities owing to its less electronegativity when compared to regular peptides. ${ }^{[18,19]}$ The same is expected in the case of UP derivatives. ${ }^{[20]}$ However their (UP, TUP, and SUP) applications have not been explored to a greater extent because of the complexity in their synthesis. ${ }^{[21,22]}$ Nevertheless, an overview of their electronic, absorption, and charge transfer properties could highlight their versatile applications in various fields like photoswitches and photosensors. In this regard, we have studied the electronic structures at ground and excited states along with absorption properties. Further, the significant contribution of thio and SUPs in charge transduction have been studied by generating the hole at the donor and mobilizing the electron at the vertically ionized state using density functional theoretical (DFT) methods.

\section{2 | COMPUTATIONAL METHODS}

The models studied in the work are optimized using DFT methods as implemented in the Gaussian 09 package. ${ }^{[23]}$ The calculations are performed by the use of long range-corrected hybrid functionals like CAM-B3LYP with D3 dispersion correction, wB97XD, and MO6-HF. All electron basis set $6-31+\mathrm{G}^{* *}$ as implemented in the Gaussian 09 is used to define all atoms in this work. Conformational search to find the lowest energy conformer is performed by rotating the the dihedral angle $(\omega)$ from $-180^{\circ}$ to $180^{\circ}$ at ground state with a varying stepsize of $10^{\circ}$ to get 36 initial structures. The energies of conformers coming under the relative energy gap of $15 \mathrm{kcal} \cdot \mathrm{mol}^{-1}$ are compared and have been included in Table S1. The obtained lowest energy conformer has been used for further studies on electronic and excited state properties. The computed values of structural parameters using all 3 functionals mentioned above are comparable (Table S2). Hence, the results obtained for CAM-B3LYP functional is discussed in the manuscript.

The second-order harmonic vibrational studies have been performed to characterize the computed structure as minima (all real frequencies) or transition state (TS) (only 1 imaginary frequency). The initial structures for TS is determined by rotating the dihedral angle $\omega$ from $\sim 178^{\circ}$ (reactant) to $\sim 64^{\circ}$ (product) with a stepsize of $10^{\circ}$ for all models studied in this work. The highest energy point is considered as a guess structure for TS optimization, which has been performed using the Berny optimization algorithm for TS. The presence of TS is confirmed by the presence of single imaginary frequencies. Further, intrinsic reaction coordinate calculations are performed to show that the TS is indeed connected with the respective trans (reactant) and cis (product). The relative energies of TS and product with respect to the reactant along with the dihedral angles have been given in Table S3.

Excited-state calculations are performed using timedependent DFT. Optimization of the geometry at first excited state is performed to understand the geometrical change at that particular state. The CAM-B3LYP functional has also been proven to give accurate results for studying the excited states without any correlation between error and spatial orbital overlap values $(\lambda)$ for similar type of molecules. ${ }^{[24]}$ Natural population analysis ${ }^{[25]}$ have been performed to analyze the mode of charge distribution in molecules. Ionization of the moieties results in a doublet cationic state. Vertical ionization energy (VIE) is obtained as a difference in the energy of the optimized neutral $\left(\mathrm{E}_{\mathrm{N}}\right)$ and the cationic state $\left(\mathrm{E}_{\mathrm{N}-1}\right)$ after immediate ionization at neutral geometry. Adiabatic ionization energy (AIE) is given by the energy difference between a relaxed neutral state $\left(E_{N}\right)$ and a relaxed the cationic state $\left(\mathrm{E}_{\mathrm{N}-1}^{\prime}\right)$. Thus VIE and AIE can be calculated using the equations given below.

$$
\mathrm{VIE}=\left(\mathrm{E}_{\mathrm{N}}\right)-\left(\mathrm{E}_{\mathrm{N}-1}\right) \text { and } \mathrm{AIE}=\left(\mathrm{E}_{\mathrm{N}}\right)-\left(\mathrm{E}_{\mathrm{N}-1}^{\prime}\right)
$$

Hole migration is monitored via migration of a hole produced after vertical ionization (VI) of the neutral molecule. This produces a nonstationary electronic state, which evolves in time. The hole migration occurs at VI, before nuclear relaxation or at a frozen frame nuclei. The VI creates a localized hole in the highest occupied molecular orbital (HOMO) by removing the electron from the same. The details of the 
methods have been illustrated by Remacle et $\mathrm{al}^{[26]}$ and used already for similar molecules. ${ }^{[20]}$

\section{3 | RESULTS AND DISCUSSIONS}

The models studied in this work have been schematically represented in Figure 1 and have been listed in the Figure 2. The TUP and SUP models for the computations have been generated by replacing oxygen in the ureido group of UPs by sulphur and selenium, respectively. The former has been synthesized as a TUP ester, N-BocPhe- $\psi\left[\mathrm{CH}_{2} \mathrm{NCS}\right]^{[22]}$ and the later with many derivatives like methyl 2(3-cyclohexylselenoureido)-3-phenylpropanoate. ${ }^{[21]}$ The protecting groups at both carboxylate end and amino end have been modelled as $\left[-\mathrm{CH}_{3}\right]$ group to reduce computational cost. The models are named as M1, M2, M3, and M4. Each comprises of both TUP and SUP as represented in Figures 1 and 2. M1S/Se is directly inspired from the synthesized models mentioned above whereas other models support the better understanding of the same. They differ in the number of alkyl groups between the ureido and carboxylate end extending the peptide chain length. Thus, the influence and reproducibility of results can be compared with respect to the distance between donor and acceptor.<smiles>[14CH3]NC(=O)N[TlH]</smiles><smiles>[X]C(=O)NC(=O)OC([2H])(C)C(=O)OC</smiles>

Trans conformer

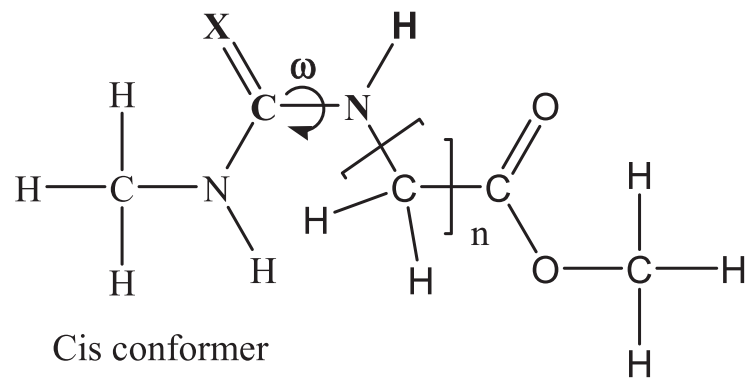

Where $X=S / S e, M 1: n=1, M 2: n=3, M 3: n=5, M 4: n=7$

FIGURE 1 Schematic representation of different models studied in the work ( $\omega$ represents the dihedral angle of the peptide bond)

\section{1 | Structural and electronic properties}

The substitution of highly polarizable $\mathrm{S}$ and Se results in a few structural features in general when compared to UPs. Computed structural parameters are comparable with experimental results (Table S2) and the corresponding vibrational normal modes are given in Table 1. The change in normal mode is in direct correlation with bond length changes. Increase in bond length decreases the stretching frequencies and vice versa. For example, the $v_{\mathrm{C}-\mathrm{S} / \mathrm{C}-\mathrm{Se}}$ is decreased by 250 to $300 \mathrm{~cm}^{-1}$ compared to $v_{\mathrm{C}-\mathrm{O}}$, which is a direct consequence of increase in bond length.

Computation shows the following trend in energetics when compared to UPs.

1. Irrespective of the substitution, lowest energy conformers of TUPs and SUPs prefer trans conformation with respect to ureido $\mathrm{C}-\mathrm{N}$ bond $(\omega)$ as reported for UPs.

2. Similar to UPs, both M1S and M1Se with a shorter peptide chain length exist as a linear conformer in the ground state (Figure 2).

3. M2S has a stable lowest energy conformer with a linear geometry, while M2Se prefers a distinct bent conformer (stabilized by $1.5 \mathrm{eV}$ ). This might be due to the electrostatic interaction between electropositive selenium atom and the electronegative carboxylate group that comes under the range of $2.911 \AA$. However, there is no sign of an intramolecular hydrogen bonding interaction, since all the hydrogen atoms present are beyond the distance possible for such an interaction.

4. Unlike, oxo peptides, the less polarized $\mathrm{S}$ and Se containing peptide do not possess the direct strong $\mathrm{H}$-bond within peptide bond (between $\mathrm{C}=\mathrm{X}$ and $\mathrm{NH}$ unit). However, the intramolecular hydrogen bonding interaction exists between the peptide group and adjacent groups (Figure S1). This in turn leads to some structural peculiarities. In M1 and M2, H-bonding leads to the stabilization of structures that align the alkyl groups in the peptide chain at an angle of $120^{\circ}$. Presence of H-bond between $\mathrm{H}(28)$ and $\mathrm{O}$ (14) in M3 and M4 models induces the formation of a 6-membered pseudocycle at the carboxylate end. The trend remains the same in case of excited-state geometries.

5. The longer chain models (M3S, M3Se, M4S, M4Se along with M2S) prefer linear geometry at the ground state, which is contradictory to that reported for similar longer chain UPs. ${ }^{[20]}$ The increase in chain length (M3 and M4) does not give way for folded conformers, hence both the models prefer linear geometry because it is the lowest energy conformer. Various structural attributes of these lowest energy conformers are further compared with the reported structural parameters of the respective UP models. The computed/experimental ${ }^{[17]} \mathrm{C}-\mathrm{S}$ (1.67/1.68)

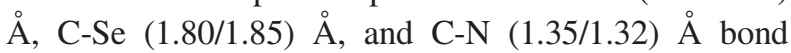




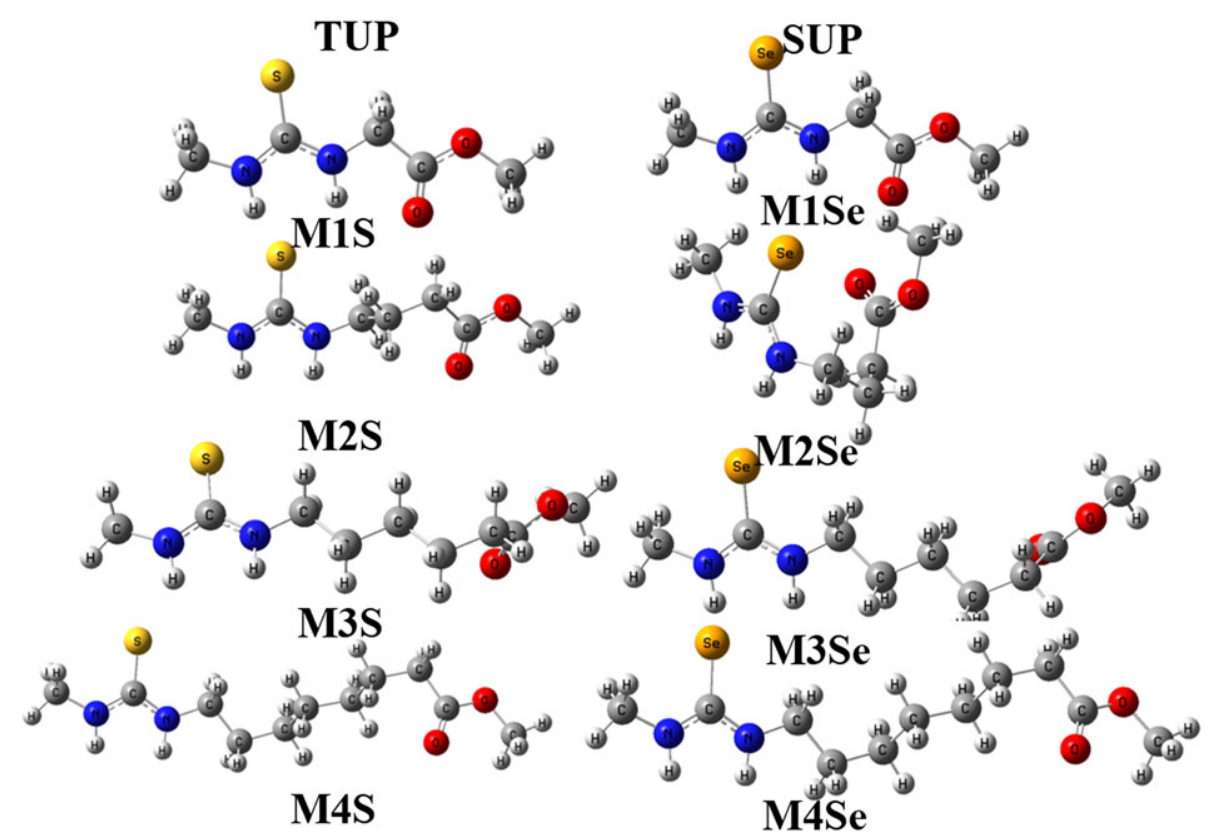

FIGURE 2 Optimized structures of different TUP and SUP models studied in the work using CAM-B3LYP functional. SUP, selenoureidopeptide; TUP, thioureidopeptide

TABLE 1 Characteristic vibrational frequencies $\left(\mathrm{cm}^{-1}\right)$ of different TUP and SUP models in both trans and cis (in italics) geometries are studied using CAM-B3LYP functional

\begin{tabular}{|c|c|c|c|c|c|}
\hline Models & & $\mathbf{v}_{\mathbf{C}-\mathrm{X}}$ (ureido end) & $\mathbf{v}_{\mathbf{C}-\mathbf{N}}$ & $\mathbf{v}_{\mathrm{N}-\mathrm{H}}$ & $\mathbf{v}_{\mathbf{C}-\mathrm{O}}($ carboxylate end $)$ \\
\hline \multirow{5}{*}{ TUPs } & & 1293 & 1524 & 3589 & 1847 \\
\hline & & 1233 & 1505 & 3465 & 1820 \\
\hline & M3S & 1481 & 1590 & 3637 & 1833 \\
\hline & & 1250 & 1575 & 3668 & 1829 \\
\hline & & 1320 & 1533 & 3443 & 1834 \\
\hline \multirow[t]{4}{*}{ SUPs } & M1Se & 1396 & 1695 & 3595 & 1810 \\
\hline & & 1303 & 1584 & 3569 & 1848 \\
\hline & $\mathrm{M} 2 \mathrm{Se}$ & 1390 & 1596 & 3619 & 1827 \\
\hline & & 1315 & 1537 & 3428 & 1818 \\
\hline
\end{tabular}

Abbreviations: SUP, selenoureidopeptide; TUP, thioureidopeptide.

distances are corroborated well with each other. Further, the shift in the values of important structural parameters under the influence of $\mathrm{S}$ and Se substitution in ureido units are examined, which led to the following observations:

6. C-Se (1.80 $\AA$ ) bond length in SUP is greater than that of C-S $(1.67 \AA)$ in TUP in turn than C-O (1.21 $\AA$ ) in UP, which is correlated to the decrease in electronegativity and increase in ionic size down the group.
7. Substitution of oxygen atom with its less electronegative $\mathrm{S} / \mathrm{Se}$ counterparts shorten the adjacent $\mathrm{C}-\mathrm{N}$ bond (1.34-1.35) $\AA$ compare to that in UPs $(1.37-1.42) \AA$.

Further, Natural Population Analysis (NPA) predicts the natural negative charge localization on thio and seleno ureido groups (Table 2). Thus, both thio and selenoureido groups can be electron donors and the respective carboxylate centers 
TABLE 2 Computed charges from NPA at the ureido and carboxylate ends of different TUP and SUP models studied using CAM-B3LYP functional

\begin{tabular}{llcc} 
Models & & Ureido End & Carboxylate End \\
\hline \multirow{2}{*}{ TUPs } & M1S & -0.215 & 0.007 \\
& M2S & -0.236 & -0.009 \\
& M3S & -0.243 & -0.006 \\
\multirow{3}{*}{ SUPs } & M4S & -0.243 & -0.010 \\
& M1Se & -0.217 & 0.006 \\
& M2Se & -0.227 & 0.003 \\
& M3Se & -0.244 & -0.006 \\
& M4Se & -0.245 & -0.011 \\
\hline
\end{tabular}

The atoms involved in the analysis of charge at ureido and carboxylate end using NPA has been shown in Figure S2.

Abbreviations: SUP, selenoureidopeptide; TUP, thioureidopeptide.

can be electron acceptors. The introduction of highly polarizable $\mathrm{S}$ and Se increases the negative charge at ureido end by $\sim 0.1$ e compared to that in UPs. And the reverse is observed at carboxylate end.

The values of HOMO-LUMO gap in TUP and SUP $(7 \mathrm{eV})$ (Table 3 ) are decreased by $2 \mathrm{eV}$ compared to UP $(9 \mathrm{eV})$. This indicate that the increase in polarizability make these species chemically more reactive than UPs. To understand further, the eigenfunctions of HOMO and LUMO orbitals are compared with that of UPs (MO diagram in Figure 3). In HOMO, the lone pair of electrons are largely localized on S and Se in TUP and SUP, respectively, whereas it is delocalized over the ureido group in case of UPs. The absence of delocalization increases the energy of HOMO and as a result, decreases the HOMO-LUMO gaps. Moreover, the increase in the chain length does not alter the trend in the charge distribution or HOMO-LUMO gap (Table 3).

\section{2 | Excited-state properties}

Subsequently, electron excitation studies predict the possibility of 2 types of transitions in general, (1) The higher intense peak 1 at 200 to $250 \mathrm{~nm}$ arises because of the transitions from n-orbital (lone pair) localized at ureido end to $\pi^{*}$ orbital localized at carboxylate end. (2) The less intense peak 2 at $\sim 250$ to $300 \mathrm{~nm}$ arises because of the transition from $\pi$ orbital at ureido end to $\pi^{*}$ orbital at the carboxylate end (Figures 3 and S3). Both the peaks appear because the transition from ureido to carboxylate end.

The presence of $\mathrm{n}$ to $\pi^{*}$ transitions led us to probe the possibility for a conformational switching as in oxopeptides. ${ }^{[2]}$ Thus, the geometry has been optimized at the first excited state to understand the conformational switching. Irrespective of the differences in models, all the conformers of TUP and SUP are switched to the new conformers by a rotation of the peptide $\mathrm{N}-\mathrm{H}$ bond and changing its dihedral angle $(\omega)$ ranges from $\left(173^{\circ}-177^{\circ}\right)$ to $\left(64^{\circ}-67^{\circ}\right)$. The regular algorithm of optimization of conformers does not stabilize the cis form in models M1S and M1Se because of the shorter chain length. The cis conformer of M1S and M1Se is stabilized by freezing the rotation of $\mathrm{H}-\mathrm{N}-\mathrm{C}-\mathrm{S} / \mathrm{Se}$ plane. The restrained optimized geometry has lowester energy structures and have all real frequencies at excited state. (Table S4) However, the longer chain models M2, M3, and M4 are showing the conformational swapping during the excited state optimization (Tables S1 and S4) similar to oxopeptides. Further, the vibrational frequencies of both conformers have been analysed to highlight the structural differences. The dihedral change decreases the stretching frequencies of C-Se, C-N, and N-H of cis conformer when compared to tras, which is in correlation with bonding distances. The bond distances are more in cis conformer because of the steric repulsion compare to trans.

And note that this kind of a rotation of peptide bond is not observed for UP models. ${ }^{[20]}$ The gradual change from delocalized "n" orbital to localized ones on going down the chalcogen group could be the reason (Figure S4). Further, the rotational barriers have been computed and found to be in the range of 10 to $17 \mathrm{kcal} \cdot \mathrm{mol}^{-1}$ for all the TUP and SUP models that are lower than that reported for oxopeptides. ${ }^{[2]}$ Orbital picture reflects that transitions are preferred from the sulphur and selenium substituted ureido group to

TABLE 3 The computed HOMO-LUMO gap, VIE, AIE, and the timescale for a complete cycle of hole migration from 1 end to another are given

\begin{tabular}{llcccc} 
Models & & DLUMO-HOMO $(\mathbf{e V})$ & VIE(eV) & AIE (eV) & Timescale for 1 cycle (fs) \\
\hline \multirow{2}{*}{ TUPs } & M1S & 7.201 & 8.011 & 7.796 & 4.4 \\
& M2S & 6.812 & 7.896 & 7.676 & 3.2 \\
& M3S & 7.131 & 7.608 & 7.488 & 3.2 \\
\multirow{3}{*}{ SUPs } & M4S & 7.442 & 7.828 & 7.622 & 2.6 \\
& M1S & 6.921 & 7.622 & 7.450 & 3.1 \\
& M2S & 7.001 & 7.466 & 7.208 & 2.5 \\
& M3S & 7.213 & 7.260 & 7.124 & 2.8 \\
\hline
\end{tabular}

Abbreviations: AIE,adiabatic ionization energy; HOMO, highest occupied molecular orbital; LUMO, lowest unoccupied molecular orbital; SUP, selenoureidopeptide; TUP, thioureidopeptide. 

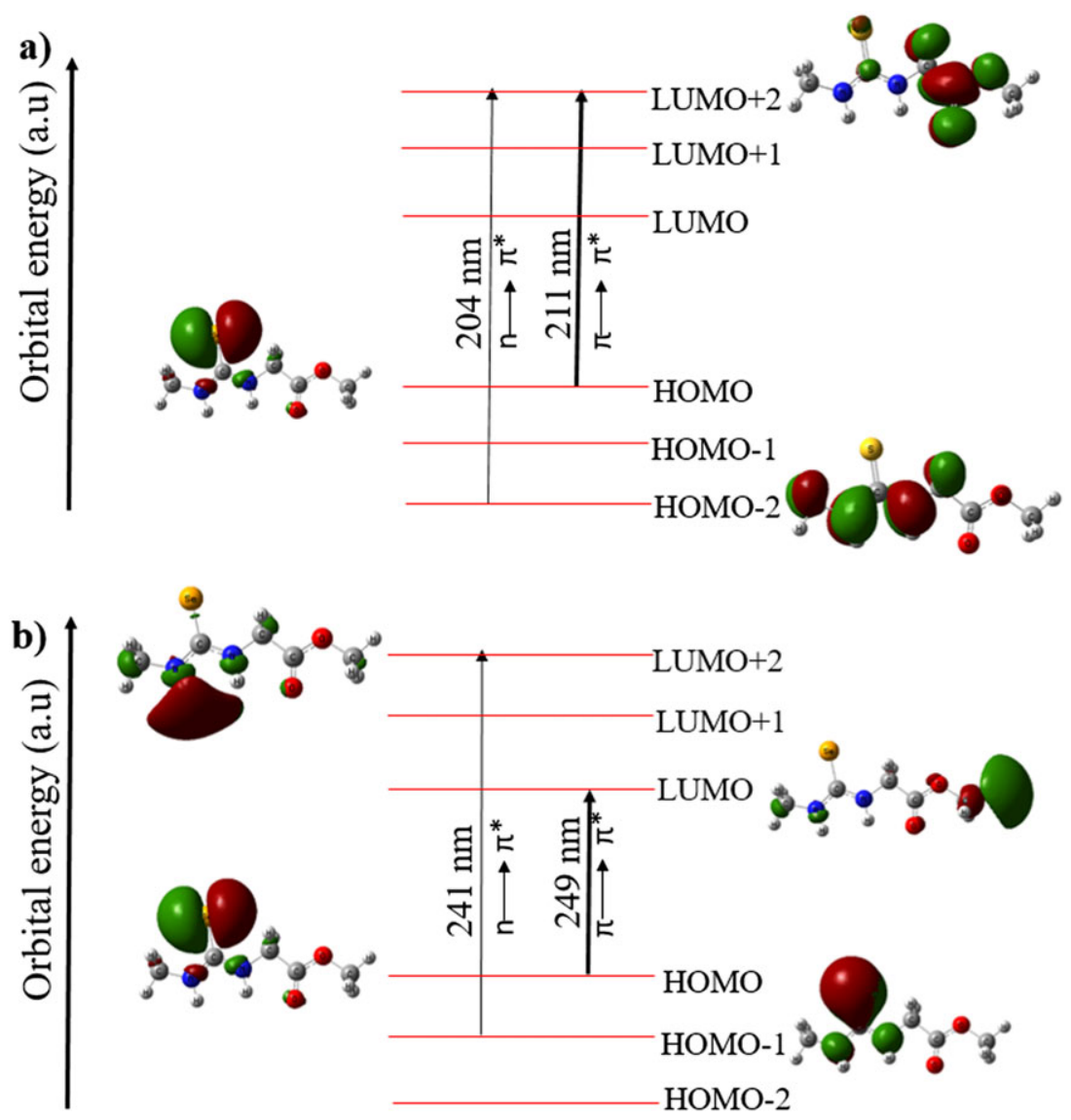

FIGURE 3 Computed excitation wavelengths of A, M1S and B, M1Se using CAM-B3LYP functional. The corresponding orbital plots are given (isovalue $=0.03 \AA^{-3}$ ) carboxylate group since HOMO orbitals are localized mostly at that end.

The wavelength range of these strong excitations gets tuned according to the presence of either sulfur or selenium at the ureido end. The peaks observed for TUPs fall between 200 to $250 \mathrm{~nm}$ and 250 to $300 \mathrm{~nm}$ for SUP, which display an evident red shift, when sulphur in ureido group is exchanged with selenium. The observation is common for all the models studied as shown in Figures 3 and S3. And Table 4 gives the complete comparison of transitions observed for different models.

\section{3 | Redox properties}

Following, redox properties are analyzed to find the possibility of their application in electrochemically driven devices. The computed VIE (7-8 eV) and AIE $(7 \mathrm{eV})$ shows minimal variation in TUPs and SUPs pointing the negligible structural change associated with relaxation of cationic structures (Table 3). This is reflected in the comparison of structural parameters of optimized neutral and cationic geometries like bond length of C-S (1.67/1.64) $\AA$, C-Se (1.81/1.85) $\AA$, and
TABLE 4 Computed excitation wavelengths ( $\mathrm{nm}$ ) of different models studied using TDDFT method showing 2 types of transitions

\begin{tabular}{llcc}
\multirow{2}{*}{ Models } & & \multicolumn{2}{c}{ Transition wavelengths $(\mathbf{n m})$} \\
\cline { 3 - 4 } TUPs & & n to $\boldsymbol{\pi}^{*}$ & $\boldsymbol{\pi}$ to $\boldsymbol{\pi}^{*}$ \\
\hline \multirow{5}{*}{ SUPs } & M1S & 204 & 211 \\
& M2S & 226 & 280 \\
& M3S & 208 & 234 \\
& M4S & 207 & 237 \\
& M1Se & 241 & 249 \\
& M2Se & 230 & 270 \\
& M3Se & 224 & 231 \\
& M4Se & 230 & 252 \\
\hline
\end{tabular}

Abbreviations: SUP, selenoureidopeptide; TDDFT, time-dependent density functional theory; TUP, thioureidopeptide.

C-N (1.35/1.32) A. Moreover, spin density plots display intriguing tendencies (Figure 4).

In case of TUPs and SUPs, spin density is substantially found localized on the $\mathrm{S}$ and $\mathrm{Se}$ atoms of thio or selenoureido moieties at ureido end as evident from the spin density plots. However, in UPs, it is not localized 
around oxygen atom, and it is strongly delocalized over the ureido end (Figure 4). The computed AIE and VIE show that the possibility for nuclear relaxation soon after ionization is less, hence the hole migration dynamics is found to occur after immediate ionization of neutral molecule. This can give an insight into the charge transduction ability of the peptide moieties under consideration. The hole migration studies after ionization have been performed by propagating the HOMO and HOMO-1 orbitals of neutral on the delocalized cation orbitals.

For M1S and M1Se, the hole generated at HOMO (lone pair of electrons on $\mathrm{S} / \mathrm{Se}$ ) is not found to evolve with time, because of its localized nature. However, spin density plots of TUP and SUP display the reasonable contribution of other atoms at ureido end in this regard. Hence, the hole has been also generated at HOMO-1 and propagated to see the possibility of charge transduction. The timescale for the whole process is calculated for different models. M1S shows the hole migration from thioureido end, which reaches the other end in 1.8 femtoseconds and starts coming back in
3.6 femtoseconds and reaches the initial point in 4.4 femtoseconds (Figure S5). Whereas in M1Se, the hole reaches carboxylate end beginning from selenoureido end in 0.7 femtosecond. It stays there till 2 femtoseconds and starts coming back and reaches the ureido end in 2.3 femtoseconds, which is found to be faster when compared to the M1S. The hole created at the LUMO, at carboxylate end, is not found to evolve with time (Figure S6).

For this phenomenon to be apprehended, the similar type of studies have been performed for long chain peptides with 3, 5, 7, $\mathrm{CH}_{2}$ linker groups (M2, M3, and M4). The hole has been generated at both HOMO and HOMO-1. Interestingly, the hole generated at HOMO migrates from ureido to carboxylate end within $\sim 1$ to 2 femtoseconds. This clearly indicates that the significance of covalent-bonded peptide chain in mobilizing the electron from 1 end to another. If lone pair on the $\mathrm{S}$ and $\mathrm{Se}$ atoms has to migrate, then it should occur through space not via covalently linked peptide chain. Compared to TUPs, hole migration in SUPs are found to occur around 1 femtosecond faster. With a small difference in the

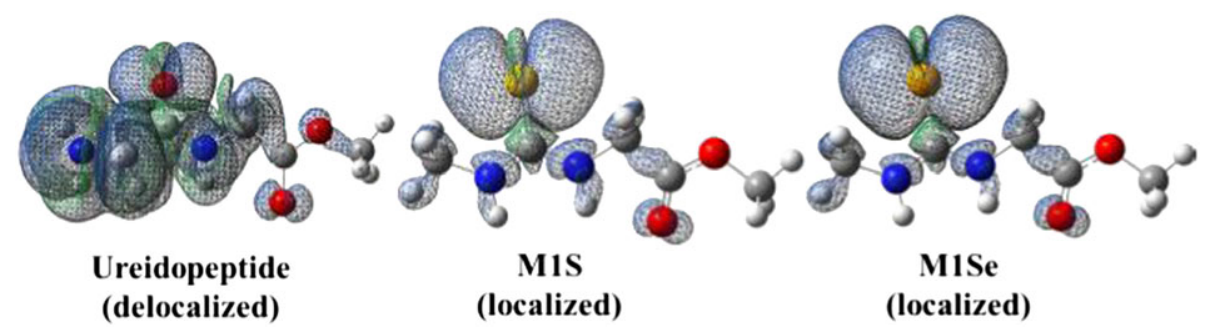

FIGURE 4 A comparison of spin density plots of ureidopeptide, M1S and M1Se showing the density profoundly located on S and Se atoms in M1S/Se. (Densities are plotted with an isocontour value of $0.002 \AA^{-3}$ )

FIGURE 5 Snapshots of the evolution of hole density from the ureido end of $\mathrm{M} 2 \mathrm{~S}$, which migrates back in $3.2 \mathrm{fs}$ (densities are plotted with an isocontour value of $0.002 \AA^{-3}$ )
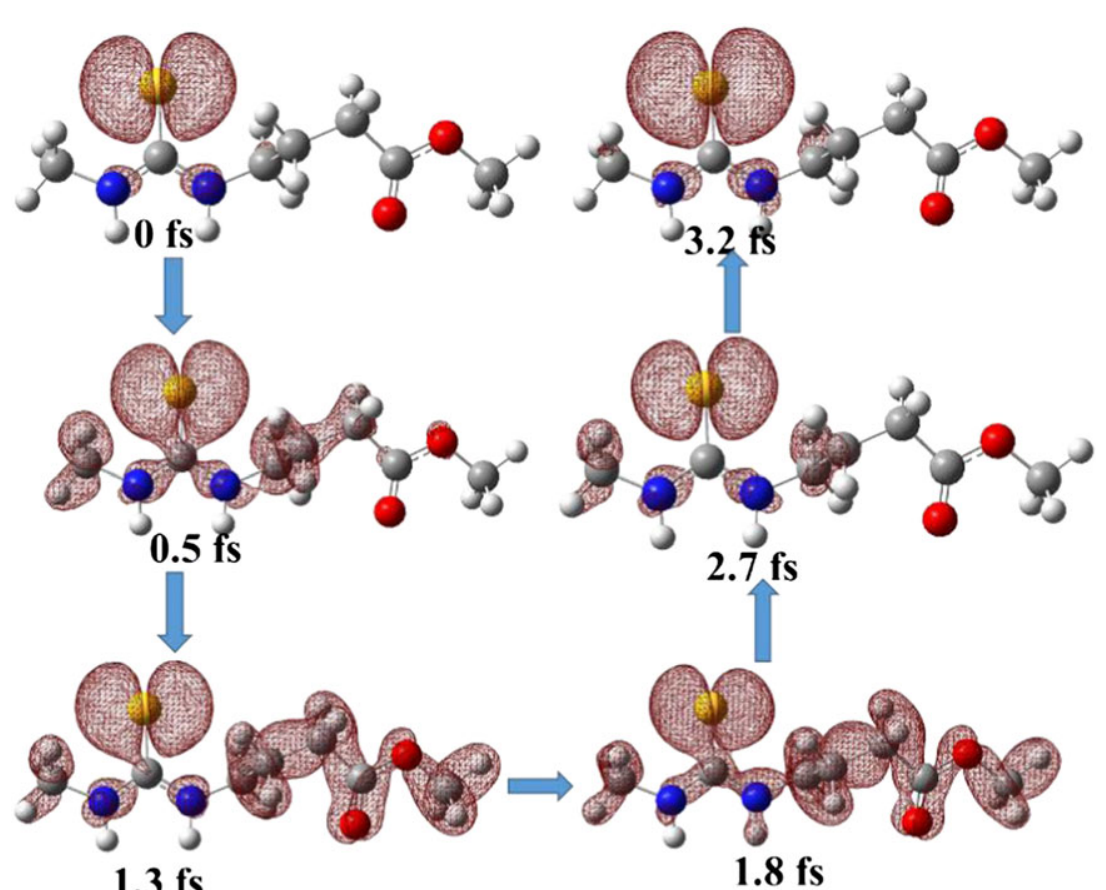
timescale, the pattern of hole migration observed in the models are same. In case of M2S (Figure 5). the hole formed at the thioureido end gets transferred to the carboxylate end in 1.8 femtoseconds and reaches the initial point back in 3.2 femtoseconds. The timescale for hole migration displayed by other models are included in Table 3 . However, none of the models studied here show the hole migration from carboxylate end.

\section{4 | CONCLUSIONS}

Our computations on different TUP and SUP models of UPs show that these entities are less prone to produce folded conformers unlike UPs. The NPA orbital and spin density plots show that charge is localized profoundly at $\mathrm{S} / \mathrm{Se}$ in case of TUP and SUP. This can make -NHCXNH- group as better donors of electrons than in UPs. Excitation studies using time-dependent DFT shows an evident red shift of SUP excitation wavelength because of the increased delocalization of electrons from that of TUPs. And the optimization of geometry at first excited state demonstrates their photoswitching ability with comparatively less-rotational barriers for all models (M1, M2, M3, and M4). Further, the spin density plots give the evidence that after ionization, electron is removed from the ureido end in TUP and SUP, where it is predominately localized at sulphur and selenium center. This is unlike that in UPs where spin density is delocalized on the complete ureido group. Hole migration dynamics of different molecules predict that covalently linked atoms in the peptide chain is necessary for charge migration. For longer chain SUP and TUP, the hole migrate at a faster timescale compared to UPs. Our studies conclude that with the isosteric substitution of UP bond with S and Se tunes the electronic structure of these moieties in such a way that they have improved specificity that can be exploited for their use as photosensors and peptide drugs.

\section{ACKNOWLEDGEMENTS}

We thank the UGC-Major Research Project (UGC-MRP), DST-SERB, Junior Research Fellowship (JRF), and Faculty Recharge Programme (FRP) for funding.

\section{REFERENCES}

[1] D. Pearson, A. D. Abell, Org BiomolChem. 2006, 4, 3618.

[2] Y. Huang, G. Jahreis, C. Lucke, D. Wildemann, G. Fischer, J. Am. Chem. Soc. 2010, 132, 7578.

[3] S. Liu, M. Chen, J. D. Li, H. B. Zhang, J. Q. Wang, S. R. Yang, Sci China Ser B Chem. 2009, 52, 1009.

[4] S. Kitzig, M. Thilemann, T. Cordes, K. Ruck-Braun, ChemPhysChem. 2016, 17, 1252.
[5] A. A. Beharry, G. A. Woolley, Chem. SocRev. 2011, 40, 4422.

[6] O. I. Lungu, R. A. Hallett, E. J. Choi, M. J. Aiken, K. M. Hahn, B. Kuhlman, Chem. Biol. 2012, 19, 507.

[7] Y. Huang, Z. Y. Cong, L. F. Yang, S. L. Dong, J. Pept. Sci. 2008, 14,1062 .

[8] G. Prabhu, N. Narendra, Basavaprabhu, V. Panduranga, V. V. Sureshbabu RSC Adv. 2015, 5, 48331-48362.

[9] V. Semetey, C. Hemmerlin, C. Didierjean, A. P. Schaffner, A. G. Giner, A. Aubry, J. P. Briand, M. Marraud, G. Guichard, Org. Lett. 2001, 3, 3843.

[10] L. Fischer, V. Semetey, J. M. Lozano, A. P. Schaffner, J. P. Briand, C. Didierjean, G. Guichard, Eur. J. Org. Chem. 2007, 3944.

[11] A. Boeijen, R. M. J. Liskamp, Eur. J. Org. Chem. 1999, 2127.

[12] A. Devadoss, P. Sudhagar, C. Terashima, K. Nakata, A. Fujishima, J. Photochem. Photobiol. C-Photochem Rev. 2015, 24, 43.

[13] J. Lapic, S. Djakovic, M. Cetina, K. Heinze, V. Rapic, Eur. J. Inorg. Chem. 2010, 106.

[14] A. M. Rincon, P. Prados, J. de Mendoza, J. Am. Chem. Soc. 2001, $123,3493$.

[15] M. D. Esrafili, J. Beheshtian, N. L. Hadipour, Int. J. Quantum Chem. 2011, 111, 3184.

[16] V. V. S. Babu, N. S. Sudarshan, S. A. Naik, Int. J. Pept. Res. Ther. 2008, 14, 105.

[17] Y. R. Nelli, S. Antunes, A. Salaun, E. Thinon, S. Massip, B. Kauffmann, C. Douat, G. Guichard, Chem.-Eur. J. 2015, 21, 2870 .

[18] E. E. Alberto, V. do Nascimento, A. L. Braga, J. Braz. Chem. Soc. 2010, 21, 2032.

[19] R. Pal, G. Nagendra, M. Samarasimhareddy, V. V. Sureshbabu, T. N. G. Row, Chem. Commun. 2015, 51, 933.

[20] S. Joy, V. V. Sureshbabu, G. Periyasamy, J. Phys. Chem. B. 2016, 120,6469

[21] H. P. Hemantha, V. V. Sureshbabu, J. Pept. Sci. 2010, 16, 644.

[22] V. V. Sureshbabu, S. A. Naik, H. P. Hemantha, N. Narendra, U. Das, T. N. G. Row, J. Org. Chem. 2009, 74, 5260.

[23] M. J. Frisch, G. Trucks, W. H. B. Schlegel, G. E. Scuseria, M. A. Robb, J. R. Cheeseman, G. Scalmani, V. Barone, B. Mennucci, G. A. Petersson, H. Nakatsuji, M. Caricato, X. Li, H. P. Hratchian, A. F. Izmaylov, J. Bloino, G. Zheng, J. L. Sonnenberg, M. Hada, M. Ehara, K. Toyota, R. Fukuda, J. Hasegawa, M. Ishida, T. Nakajima, Y. Honda, O. Kitao, H. Nakai, T. Vreven, J. A. Montgomery, J. J. E. Peralta, F. Ogliaro, M. Bearpark, J. J. Heyd, E. Brothers, K. N. Kudin, V. N. Staroverov, R. Kobayashi, J. Normand, K. Raghavachari, A. Rendell, J. C. Burant, S. S. Iyengar, J. Tomasi, M. Cossi, N. Rega, J. M. Millam, M. Klene, J. E. Knox, J. B. Cross, V. Bakken, C. Adamo, J. Jaramillo, R. Gomperts, R. E. Stratmann, O. Yazyev, A. J. Austin, R. Cammi, C. Pomelli, J. W. Ochterski, R. L. Martin, K. Morokuma, V. G. Zakrzewski, G. A. Voth, P. Salvador, J. J. Dannenberg, S. Dapprich, A. D. Daniels, Ö. Farkas, J. B. Foresman, J. V. Ortiz, J. Cioslowski, D. J. Fox, Gaussian 09, Revision D.01, Gaussian, Inc., Wallingford.

[24] M. J. G. Peach, P. Benfield, T. Helgaker, D. J. Tozer, J. Chem. Phys. 2008, 128. 
[25] J. P. Foster, F. Weinhold, J. Am0 ChemSoc. 1980, 102, 7211.

[26] F. Remacle, R. D. Levine, Proc. Natll. Acad. Sci. U S A. 2006, 103, 6793.

\section{SUPPORTING INFORMATION}

Additional Supporting Information may be found online in the supporting information tab for this article.
How to cite this article: Joy S, Sureshbabu VV, Periyasamy G. Density functional theoretical studies on photoswitching and charge migration dynamics of thio and selenoureidopeptides. J Phys Org Chem. 2017;e3693. https://doi.org/10.1002/poc.3693 